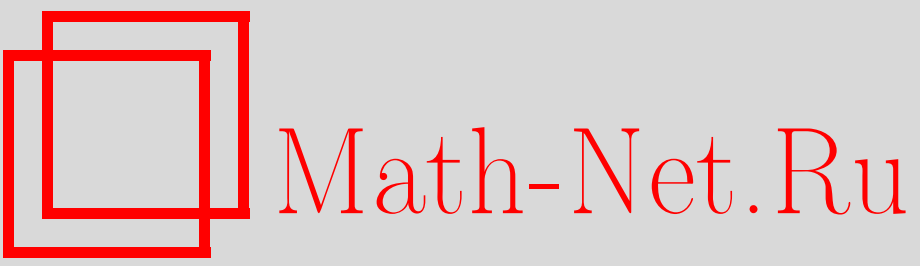

Ю. А. Кашлев, Неравновесная статистическая термодинамика каскадных процессов в твердых телах: квазитемпература каскадных частиц, ТМФ, 2001, том 126, номер 2, 311-324

DOI: https://doi.org/10.4213/tmf433

Использование Общероссийского математического портала Math-Net.Ru подразумевает, что вы прочитали и согласны с пользовательским соглашением

http://www.mathnet.ru/rus/agreement

Параметры загрузки:

IP : 52.90 .164 .192

26 апреля 2023 г., 13:00:15 


\section{НЕРАВНОВЕСНАЯ СТАТИСТИЧЕСКАЯ ТЕРМОДИНАМИКА КАСКАДНЫХ ПРОЦЕССОВ В ТВЕРДЫХ ТЕЛАХ:} КВАЗИТЕМПЕРАТУРА КАСКАДНЫХ ЧАСТИЦ

В рамках неравновесной статистической термодинамики исследованы каскады смещений в твердых телах. С использованием уравнения баланса энергии в условиях каскадного процесса дан вывод квазитемпературы подсистемы каскадных частиц в металлах и полупроводниках.

\section{1. УРАВНЕНИЕ БАЛАНСА ЭНЕРГИИ КАСКАДНЫХ ЧАСТИЦ В ТВЕРДЫХ ТЕЛАХ}

Уравнение баланса энергии каскадных частиц, лежащее в основе данного исследования, рассматривалось в работе [1].

Понятие квазитемпературы быстрых частиц, значительно превьшаюшей температуру термостата, вводилось при изучении ряда физических явлений. Оно использовалось при анализе охлаждения пучка быстрых протонов в электронном газе [2] и термализации релятивистских позитронов в твердых телах [3], [4], в теории движения быстрых электронов в полупроводнике под воздействием электрического поля [5], а также в теории "разогрева" высокоэнергетических атомных частищ, движушихся в кристалле в режиме каналирования [6]. Продолжением исследований является данная работа, в которой развита неравновесная статистическая термодинамика подсистемы выбитых из узла (движушихся) атомов, образовавшихся в твердом теле в условиях каскада смешений. Основное внимание уделяется анализу квазитемпературы $1 / F_{21}$ подсистемы выбитых атомов (в дальнейшем - каскадных частиг).

В качестве отправной точки исследования принимается уравнение баланса энергии каскадных частиц (КЧ) в квазиравновесном состоянии или в непосредственной близости от него [1]

$$
\frac{d}{d t} \bar{E}_{2}=\bar{\varepsilon} f_{3}\left(F_{21}\right)\left(\frac{d N_{2}}{d t}\right)-L_{\dot{H}_{2} \dot{H}_{2}}(X) X
$$

\footnotetext{
* Институт металлургии и материаловедения им. А.А. Байкова РАН, Москва, Россия
} 
Здесь $\bar{E}_{2}$ - внутренняя энергия подсистемы КЧ, первый член в правой части (1) - мощность, вводимая в подсистему КЧ внешним излучением, второй член описывает отвод энергии из каскадной области. Средняя энергия, вводимая в единицу времени, включает среднюю энергию КЧ в предельном случае $1 / F_{21} \rightarrow \infty$

$$
\bar{\varepsilon}=C_{2} \frac{\pi}{2} \frac{1}{\alpha},
$$

скорость размножения КЧ $d N_{2} / d t$ и функцию обратной квазитемпературы КЧ $F_{21}$, имеющую вид

$$
f_{3}\left(F_{21}\right)=2\left[\varphi\left(F_{21}\right)\right]^{-2}\left\{1-\left[1+\varphi\left(F_{21}\right)\right] \exp \left(-\varphi\left(F_{21}\right)\right)\right\}
$$

где

$$
\varphi\left(F_{21}\right)=\frac{\pi}{2} F_{21} \stackrel{*}{\varepsilon}
$$

и использованы следуюшие обозначения: $\alpha=2 M R^{2}, M$ - масса атома решетки, $R$ параметр потенциала Борна-Майера $[1], \stackrel{*}{\varepsilon}=(12)^{1 / 3}(1 / \alpha)$. Величины $C_{i}$ - численные коэффициенты, коэффициент $C_{2}$ будем рассматривать как свободный параметр теории. Полная скорость образования КЧ в уравнении (1)

$$
\frac{d N_{2}}{d t}=k_{1} N_{1}, \quad k_{1}=\widetilde{\Phi} \sigma^{(\mathrm{d})} \bar{\nu}\left[1-\frac{8}{3} \sigma^{(\mathrm{r})} N_{\mathrm{V}} a\right]
$$

включает $\widetilde{\Phi}$ - плотность потока бомбардируюших частиц, $\sigma^{(\mathrm{d})}$ - поперечное сечение столкновений, приводяших к смешению атомов решетки, $\bar{\nu}-$ каскадную функцию, усредненную по энергетическому спектру первично выбитых атомов, $N_{1}$ - плотность числа атомов решетки. Второй член в квадратных скобках в выражении для $k_{1}$ в $(3)$ описывает вклад спонтанной атермической рекомбинации [1], и в нем $\sigma^{(\mathrm{r})}-$ сечение рекомбинации, $N_{\mathrm{V}}$ - плотность термодинамически неравновесных вакансий, образовавшихся в процессе облучения, $a$ - параметр решетки. (Здесь и далее используется система единищ, в которой $\hbar=k_{\mathrm{B}}=1$.)

Поскольку в условиях каскадного процесса система, состояшая из подсистемы КЧ и термостата, сильно отклоняется от термодинамического равновесия, отвод энергии в уравнении (1) описывается с помощью нелинейной диссипативной функции. Поэтому коэффициент переноса $L_{\dot{H}_{2} \dot{H}_{2}}(X)$ зависит от термодинамической силы $X=\beta_{1}-F_{21}$, в которой $\beta_{1}=1 / T_{1}$ - обратная температура термостата. Этот коэффициент, в свою очередь, может быть выражен через коэффициент $L_{\dot{H}_{2} \dot{H}_{2}}\left(F_{21}\right)$, входящий в линейное соотношение между потоком энергии и термодинамической силой,

$$
L_{\dot{H}_{2} \dot{H}_{2}}(X)=L_{\dot{H}_{2} \dot{H}_{2}}\left(F_{21}\right) f_{4}\left(\frac{\bar{\omega}}{2} X\right) \text {, }
$$

где $f_{4}(y)=\operatorname{th} y / y, \bar{\omega}-$ среднее значение переданной энергии в случае подпороговых столкновений, характерных для релаксационного этапа каскада смещений. Расчет показал [1], что в борновском приближении коэффициент переноса $L_{\dot{H}_{2} \dot{H}_{2}}\left(F_{21}\right)$ можно выразить через вероятность перехода в единицу времени $P_{\mu \nu, \mu^{\prime} \nu^{\prime}}$. А именно,

$$
L_{\dot{H}_{2} \dot{H}_{2}}\left(F_{21}\right)=\frac{1}{2} \sum_{\mu \mu^{\prime} \nu \nu^{\prime}}\left(\varepsilon_{\nu}-\varepsilon_{\nu^{\prime}}\right)^{2} P_{\mu \nu, \mu^{\prime} \nu^{\prime}} n^{(2)}\left(\varepsilon_{\nu}\right) n_{\mu}^{(1)},
$$


где $\varepsilon_{\nu}$ - энергия КЧ, $n_{\mu}^{(1)}$ и $n^{(2)}\left(\varepsilon_{\nu}\right)$ - средние числа заполнения состояния термостата и состояния подсистемы КЧ, причем $n^{(2)}\left(\varepsilon_{\nu}\right)=\lambda \exp \left(-F_{21} \varepsilon_{\nu}\right)$, где $\lambda$ - абсолютная активность КЧ [1], [7].

В условиях сильно неравновесной системы введение квазитемпературы в качестве модуля экспоненшиального распределения одной из ее подсистем (в приближении малой плотности частиц) возможно либо при наличии взаимодействия между частицами подсистемы (или внутри какой-либо степени свободы), либо при взаимодействии некоторых степеней свободы с термостатом. Первый случай характерен для спин-фононных систем, когда спиновая подсистема за счет взаимодействия переходит в состояние внутреннего равновесия с больцмановским распределением заселенности [8]. Второй случай реализуется в плазме, а также в условиях каналирования быстрых частиц в кристаллах [6], [9]. В этих работах рассматривается обмен поперечных степеней свободы движения частиц с термостатом, что представляет большой интерес с точки зрения ситуации каскадообразования. Обмен приводит к установлению поперечной квазитемпературы каналированных частиц на глубине порядка длины когерентности. Причем в случае каналирования [6] неупругое электронное рассеяние частиц приводит к разбросу поперечной энергии каналированных частиц, который, в свою очередь, определяет модуль распределения частищ, т.е. квазитемпературу. Аналогично, рассматривая КЧ как самостоятельную термодинамическую подсистему, мы установили связь квазитемпературы КЧ с фолуктуацией их энергии [1]

$$
\frac{1}{F_{21}(t)}=\left\{\frac{1}{C_{V}}\left(H_{2}, H_{2}\right)^{t}\right\}^{\frac{1}{2}}
$$

Здесь $C_{V}$ - теплоемкость одноатомного газа КЧ, $H_{2}$ - гамильтониан КЧ, $\left(H_{2}, H_{2}\right)^{t}-$ временная корреляционная функция.

В данной работе с помощью уравнения баланса (1) устанавливается связь коэффициента переноса (5) с собственным значением матрицы релаксации. Это собственное значение используется для расчета дисперсии процесса в рамках флуктуационно-диссипативной теории, а затем на основе соотношения (6) выгисляется квазитемпература KU.

\section{2. ОБЩИЙ ВИД КОЭФФИЦИЕНТА ПЕРЕНОСА В УСЛОВИЯХ ЭЛЕКТРОННОГО РАССЕЯНИЯ КЧ (МАЛЫЕ СКОРОСТИ ЧАСТИЦ, МАЛЫЕ ПЕРЕДАЧИ ИМПУЛЬСА)}

Будем рассматривать диссипативный процесс за счет возбуждения электронов вблизи поверхности Ферми (в дальнейшем энергия Ферми обозначается $E_{\mathrm{F}}$ ). Для этого введем двухвременную запаздываюшую функцию Грина

$$
G(\mathbf{q}, t)=-i \Theta(t)\left\langle\left[\rho_{\mathbf{q}}(t), \stackrel{+}{\rho}_{\mathbf{q}}(0)\right]\right\rangle_{\mathrm{eq}},
$$

включающую $\rho_{\mathbf{q}}-$ фурье-компоненту флуктуации плотности электронов. В формуле $(7)\langle\ldots\rangle$ еq означает усреднение по равновесному распределению электронов, $\Theta(t)=1$ 
при $t>0$. Функция Грина плотность - плотность нулевого порядка $G^{(0)}(\mathbf{q}, t)$ также может быть записана в форме (7), если ввести усреднение по ансамблю невзаимодействующих электронов.

Вероятность перехода задается соотношением

$$
\mathcal{P}(\mathbf{q}, \omega) d \mathbf{q} d \omega=-\frac{1}{(2 \pi)^{2}}|V(q)|^{2} S(\mathbf{q}, \omega) \delta((\mathbf{q v}(\bar{\varepsilon}))-\omega) d \mathbf{q} d \omega
$$

где динамический форм-фактор $S(\mathbf{q}, \omega)$ выражается через фурье-компоненту (по временной переменной) функции Грина (7)

$$
S(\mathbf{q}, \omega)=\frac{1}{\pi} \operatorname{Im}\left\langle\left\langle\rho_{\mathbf{q}} \mid \stackrel{+}{\rho}_{\mathbf{q}}\right\rangle\right\rangle_{\omega}
$$

В (8) использованы обозначения: $V(q)=\left(4 \pi Z_{1} e^{2} / q^{2}\right), Z_{1}$ - атомный номер КЧ, $v(\bar{\varepsilon})=$ $k_{v} \sqrt{\bar{\varepsilon}}-$ скорость КЧ.

Большинство статистических интерпретаций каскадов дается в рамках статистической механистической теории, когда средние числа заполнения состояний КЧ являются постоянными, что соответствует пределу $\left(1 / F_{21}\right) \rightarrow \infty[6]$. В этом случае $\lambda=1[1]$, так что коэффициент переноса (5) принимает более простой вид

$$
\tilde{L}_{\dot{H}_{2} \dot{H}_{2}}=\lim _{\frac{1}{F_{21}} \rightarrow \infty} L_{\dot{H}_{2} \dot{H}_{2}}\left(F_{21}\right)=\frac{1}{2} \int_{-\infty}^{+\infty} d \omega \omega^{2} \int d \mathbf{q} \mathcal{P}(\mathbf{q}, \omega) .
$$

Подставляя (8) в (9) и интегрируя по угловым переменным вектора $\mathbf{q}$, получаем

$$
\begin{aligned}
\tilde{L}_{\dot{H}_{2} \dot{H}_{2}} & =i(2 \pi)^{-3} v(\bar{\varepsilon}) \frac{1}{2} \int d q q^{3}|V(q)|^{2} R(q), \\
R(q) & =\int_{-\infty}^{\infty} d \omega \omega\left\{\operatorname{Im} G(q, \omega) I_{1}(q, \omega)\right\}, \\
I_{1}(q, \omega) & =\left|\int_{-\infty}^{\infty} d \tau j_{1}(q v(\bar{\varepsilon}) \tau) \exp (-i \omega \tau)\right|,
\end{aligned}
$$

где $j_{1}(x)$ - сферическая функция Бесселя.

Теперь выполним ряд преобразований коэффициента переноса. Отбрасывая температурные поправки, вынесем из-под знака интеграла в выражении (10б) множитель $\omega$ в точке $\omega=(3 / 5) E_{\mathrm{F}}$, соответствующей энергии основного состояния электронного газа без учета вклада кулоновского взаимодействия. Такая аппроксимация обеспечивает удовлетворительную точность. В этом можно убедиться, сравнивая результат прямого вычисления $R(q)(10 б)$ в первом борновском приближении $(G(q, \omega)$ заменяется на $\left.G^{(0)}(q, \omega)\right)$ с результатом, полученным с использованием указанной аппроксимации. На релаксационном этапе достаточно ограничиться рассмотрением малых скоростей КЧ [1]. В области малых скоростей функция Бесселя аппроксимируется выражением $j_{1}(q v \tau)=q v \tau / 3$. Подставим последнее выражение в формулу (10в) и проинтегрируем по $d \tau$. Получим, что

$$
I_{1}(q, \omega)=\frac{1}{3} \frac{q v}{\omega^{2}}
$$


С учетом сделанных приближений проинтегрируем функцию $R(q)(10 б)$ по $d \omega$, используя теорему вычетов. В результате получаем

$$
\tilde{L}_{\dot{H}_{2} \dot{H}_{2}}=-\left.\frac{2}{5}\left(Z_{1} e^{2}\right)^{2} v^{2}(\bar{\varepsilon}) E_{\mathrm{F}} \int d q\left\{\frac{d}{d \omega} \operatorname{Im} G(q, \omega)\right\}\right|_{\omega=0},
$$

где верхний предел интегрирования по $d q$ равен $2 k_{\mathrm{F}}, k_{\mathrm{F}}=m v_{\mathrm{F}}, v_{\mathrm{F}}-$ скорость Ферми, $m$ - масса электрона.

Для релаксационной стадии характерны малые передачи импульса. При $q \ll k_{\mathrm{F}}$ и конечных температурах электронного газа имеем

$$
\operatorname{Im} G^{(0)}(q, \omega)= \begin{cases}\frac{\omega}{2 q v_{\mathrm{F}}} \tilde{N}\left(E_{\mathrm{F}}\right) \int_{0}^{\infty} d E_{k} \frac{\partial n^{(1)}\left(E_{k}\right)}{\partial E_{k}} & \text { при } \omega<q v_{\mathrm{F}}-\frac{q^{2}}{2 m}, \\ 0 & \text { при } \omega>q v_{\mathrm{F}}+\frac{q^{2}}{2 m},\end{cases}
$$

где $E_{k}$ - энергия электрона,

$$
n^{(1)}\left(E_{k}\right)=\left\{\exp \left[\beta_{1}\left(E_{k}-\mu_{1}\right)\right]+1\right\}^{-1},
$$

$\mu_{1}$ - химический потенциал термостата, $\widetilde{N}\left(E_{\mathrm{F}}\right)$ - плотность состояний на единицу энергии и единицу объема вблизи поверхности Ферми. Что касается полной функции Грина (7), то для ее вычисления используем уравнение в приближении Хартри [10]

$$
\frac{1}{G(q, \omega)}=\frac{1}{G^{(0)}(q, \omega)}-U_{\mathrm{eff}}(q)
$$

Эффективный потенциал запишем в форме Хаббарда [11]

$$
U_{\mathrm{eff}}(q)=\widetilde{V}(q) f_{5}(q), \quad f_{5}(q)=1-q^{2}\left[2\left(q^{2}+k_{\mathrm{F}}^{2}\right)\right]^{-1},
$$

где $\widetilde{V}(q)=4 \pi e^{2} / q^{2}$. Формулы (11)-(14) дают замкнутоевыражение для функции Грина $G(q, \omega)$ в случае электронного рассеяния КЧ.

\section{3. КОЭФФИЦИЕНТ ПЕРЕНОСА В ДВУХ КОНКРЕТНЫХ МОДЕЛЯХ}

Большинство результатов, касающихся кинетических явлений в металлах и полупроводниках, получены на базе изотропной модели с простой зоной: экстремум энергии электронов при $\mathbf{k}=0$ и вырождение по спину. Будем различать два случая.

3.1. Фермиевский газ высокой плотности (металл). Если $q \ll k_{\mathrm{F}}$, то мнимая часть $\operatorname{Im} G^{(0)}(q, \omega)$ отлична от нуля при $\omega<q v_{\mathrm{F}}$. Нетрудно показать, что при таких условиях $\operatorname{Im} G^{(0)}(q, \omega) \ll \operatorname{Re} G^{(0)}(q, \omega)$. В случае электронного газа в металле температурные поправки учитьвать не будем, т.е. будем считать, что $T_{1}=0$. Тогда, принимая во внимание малый вклад $\operatorname{Im} G^{(0)}(q, \omega)$, а также соотношения (12) и (13), находим

$$
\left.\frac{d}{d \omega} \operatorname{Im} G(q, \omega)\right|_{\omega=0}= \begin{cases}-\frac{1}{2 q v_{\mathrm{F}}} \tilde{N}\left(E_{\mathrm{F}}\right)\left[1-\widetilde{V}(q) \operatorname{Re} G^{(0)}(q, 0) f_{5}(q)\right]^{-2}, & 0<q \leqslant 2 k_{\mathrm{F}}, \\ 0, & q>2 k_{\mathrm{F}},\end{cases}
$$


где

$$
\widetilde{V}(q) \operatorname{Re} G^{(0)}(q, 0)=-\frac{k_{\mathrm{FT}}^{2}}{q^{2}},
$$

$k_{\mathrm{FT}}$ - обратный радиус экранирования Томаса-Ферми. Подставим (15) в (11). С учетом соотношений $(14)$ и обозначения $\xi=\left(q / 2 k_{\mathrm{F}}\right)^{2}$ имеем

$$
\begin{aligned}
\tilde{L}_{\dot{H}_{2} \dot{H}_{2}} & =C_{3}\left(Z_{1} e^{2}\right)^{2} \bar{\varepsilon} E_{\mathrm{F}} \frac{m^{2}}{M} I_{2}(\nu), \\
I_{2}(\nu) & =\int_{\xi_{\min }}^{1} d \xi \xi\left[\xi+\nu f_{6}(\xi)\right]^{-2} .
\end{aligned}
$$

Здесь $C_{3}=\left(10 \pi^{2}\right)^{-1}, \xi_{\min }=\left(k_{\mathrm{c}} / 2 k_{\mathrm{F}}\right)^{2}, k_{\mathrm{c}}=\left(\omega_{\mathrm{pl}} / v_{\mathrm{F}}\right), \omega_{\mathrm{pl}}$ - энергия плазмона, $\nu=$ $\left(k_{\mathrm{FT}} / 2 k_{\mathrm{F}}\right)^{2}, f_{6}(\xi)=(2 \xi+1)(4 \xi+1)^{-1}$. Учитывая, что для металлов с высокой плотностью электронов $\nu<1$, проинтегрируем в формуле (16б) по $d \xi$. Это дает

$$
\begin{aligned}
& I_{2}(\nu)=\ln \left(\frac{v_{\mathrm{F}}}{e^{2}}\right)-I_{3}(\nu), \\
& I_{3}(\nu)=\ln \left[\frac{1+3 d}{3 \pi(1+\nu d)}\right]+\frac{\nu(3-\nu)}{1+\nu d}, \quad d=f_{6}\left(\xi_{\min }\right) .
\end{aligned}
$$

3.2. Больцмановский газ (невырожденный полупроводник $n$-типа). Используя $(12)$ и (13), при $\omega<q v_{\mathrm{F}}$ и $T_{1} \neq 0$ получаем

$$
\left.\frac{d}{d \omega} \operatorname{Im} G(q, \omega)\right|_{\omega=0}=\left\{\begin{aligned}
& \frac{1}{2 q v_{\mathrm{F}}} \frac{1}{\zeta_{\mathrm{c}}} \tilde{N}_{\mathrm{c}}\left[1-\widetilde{V}(q) \operatorname{Re} G^{(0)}(q, 0) f_{5}(q)\right]^{-2} \times \\
& \quad \times \int_{E_{\mathrm{G}}}^{\infty} d E_{k} \frac{\partial}{\partial E_{k}} \tilde{n}^{(1)}\left(E_{k}\right) \text { при } 0<q \leqslant 2 k_{\mathrm{F}}, \\
& 0 \text { при } q>2 k_{\mathrm{F}} .
\end{aligned}\right.
$$

Здесь $\tilde{N}_{\mathrm{c}}=2\left(2 \pi \stackrel{*}{m} T_{1}\right)^{3 / 2}$ - плотность состояний на единицу объема, $\stackrel{*}{m}-$ эффективная масса электрона, $\zeta_{\mathrm{c}}=\left|E_{\mathrm{F}}-E_{\mathrm{c}}\right|-$ уровень Ферми относительно края зоны проводимости, $E_{\mathrm{G}}$ - ширина запрещенной зоны, $\tilde{n}^{(1)}\left(E_{k}\right)=\exp \left\{-\beta_{1}\left(E_{k}-\mu_{1}\right)\right\}$. Подставим (18) в (11), затем выполним интегрирование по $d E_{k}, d q$ и $d \xi$, учитывая, что в больцмановском случае (низкая плотность электронов) $\nu>1$. Ограничиваясь рассмотрением примесных полупроводников $n$-типа, заменим в полученном выражении плотность электронов проводимости $\widetilde{N}_{\mathrm{c}} \tilde{n}^{(1)}\left(E_{\mathrm{G}}\right)$ на концентрацию доноров $N_{\mathrm{d}}$. В результате получаем

$$
\begin{aligned}
& \tilde{L}_{\dot{H}_{2} \dot{H}_{2}}=(\bar{\varepsilon})^{2} C_{4} \frac{1}{\zeta_{\mathrm{c}}}\left(\bar{\varepsilon} E_{\mathrm{F}}\right)^{\frac{1}{2}} \frac{\stackrel{*}{M}}{M} \frac{1}{\tau_{\mathrm{p}}(\bar{\varepsilon})},
\end{aligned}
$$

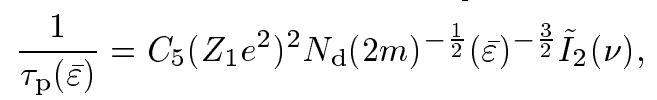

$$
\begin{aligned}
& \tilde{I}_{2}(\nu)=\ln \left(1+\frac{1}{\nu}\right)-(1+\nu)^{-1} .
\end{aligned}
$$

Здесь $C_{4}=(12 \pi / 5)=7.5, C_{5}=(1 / 16 \pi)=2 \cdot 10^{-2}, \tau_{\mathrm{p}}$ - время релаксации импульса, пропорциональное $(\bar{\varepsilon})^{3 / 2}$, что характерно для невырожденных полупроводников [12]. 


\section{4. СОБСТВЕННОЕ ЗНАЧЕНИЕ МАТРИЦЫ РЕЛАКСАЦИИ}

Для вычисления дисперсии процесса воспользуемся флуктуационно-диссипативной теорией. Уравнение баланса (1) определяет среднее поведение подсистемы КЧ на термодинамическом уровне описания. С учетом (1) стохастическое уравнение для флуктуации внутренней энергии $\delta \bar{E}_{2}$ можно записать в виде

$$
-\frac{d}{d t} \delta \bar{E}_{2}(t)+\bar{\varepsilon}\left(\frac{d N_{2}}{d t}\right) \delta f_{3}\left(F_{21}\right)=F_{21} L_{\dot{H}_{2} \dot{H}_{2}}(X) \delta\left(\frac{1}{F_{21}}\right)-\xi(t),
$$

где $\delta A(t)=A(t)-\langle A(t)\rangle-$ флуктуация. Согласно гипотезе Онзагера, любая флуктуация аналогична макроскопическому отклонению подсистемы КЧ и отличается от него только тем, что появляется спонтанно. Поэтому флуктуация удовлетворяет уравнению движения, аналогичному уравнению для среднего, но с дополнительным членом $\xi(t)$, являюшимся случайной гауссовской величиной. Именно это положение и нашло отражение в уравнении (20).

$\mathrm{C}$ точки зрения общих положений флуктуационно-диссипативной теории уравнение для флуктуации энергии (20) следовало бы дополнить уравнением для флуктуации числа КЧ $\delta \bar{N}_{2}$. Однако можно упростить теорию, если принять во внимание сушественное различие характерных времен. Дело в том что размножение КЧ происходит на каскадном этапе $\left(\sim 10^{-13} \mathrm{c}\right)$, тогда как перераспределение энергии - на релаксашионном $\left(\sim 10^{-12} \mathrm{c}\right)$. В связи с этим при изучении необратимых процессов во временной шкале переноса тепла деталями кинетики размножения можно пренебречь [1]. Поэтому уравнение для $\delta \bar{N}_{2}$ не вводится. Отметим, что та же ситуация реализуется в теории химических реакций [7], [13].

Если термодинамическая сила $X=\beta_{1}-F_{21}$ и температура решетки $T_{1}$ значительно меньше квазитемпературы "горячих" атомов $1 / F_{21}$, то вычисление ковариации случайного члена в (20) можно выполнить в полном соответствии с аналогичным расчетом из работы [13]. В этом случае ковариацию величины $\xi(t)$ удается выразить через коэффициент переноса $L_{\dot{H}_{2} \dot{H}_{2}}(X)$,

$$
\left\langle\xi(t) \xi\left(t^{\prime}\right)\right\rangle_{\mathrm{av}}=\gamma\left(F_{21}\right) \delta\left(t-t^{\prime}\right), \quad \gamma\left(F_{21}\right)=2 \eta L_{\dot{H}_{2} \dot{H}_{2}}(X), \quad \eta=\left(\frac{F_{21}}{\beta_{1}}\right)^{2} .
$$

Естественно, усреднение $\langle\ldots\rangle_{\text {av }}$ осуществляется в соответствии с принципами теории вероятностей [7]. Как следует из соотношений (21), те же самые элементарные акты, которые приводят к диссипации энергии, обусловливают одновременно и флуктуации энергии КЧ. А это, в свою очередь, отражает обший принцип флуктуационно-диссипативной теории: средние и флуктуации вызываются одними и теми же переходами частищ.

Выражая флуктуацию квазитемпературы КЧ через флуктуацию энергии

$$
\delta\left(\frac{1}{F_{21}}\right)=\frac{1}{C_{V}} \delta \bar{E}_{2},
$$


уравнение (20) можно привести к виду релаксационного уравнения [7]

$$
\frac{d}{d t} \delta \bar{E}_{2}(t)=h \delta \bar{E}_{2}(t)+\xi(t)
$$

В общем случае величина $h$ в уравнении (22) является матрицей релаксации. Однако в нашем варианте расчета, когда рассматриваются флуктуации только одной переменной, величина $h$ вырождается в скаляр. Поэтому можно рассматривать $h$ как единственное собственное значение матрицы релаксации. Принимая во внимание явный вид функции $f_{3}\left(F_{21}\right)(2 б)$, имеем

$$
\frac{\delta f_{3}\left(F_{21}\right)}{\delta\left(\frac{1}{F_{21}}\right)}=\stackrel{*}{\varepsilon} F_{21}^{2} .
$$

Учитывая эту формулу, а также формулы (20) и (22), получаем

$$
h(X)=\frac{1}{C_{V}} F_{21}^{2}\left\{\bar{\varepsilon}^{*}\left(\frac{d N_{2}}{d t}\right)-L_{\dot{H}_{2} \dot{H}_{2}}(X)\right\} .
$$

\section{5. КВАЗИТЕМПЕРАТУРА КАСКАДНЫХ ЧАСТИЦ}

В стационарном состоянии определение квазитемпературы (6) принимает болеепростОй Вид

$$
\frac{1}{F_{21}}=\left\{\frac{1}{C_{V}}\left(H_{2}, H_{2}\right)\right\}^{\frac{1}{2}},
$$

где $\left(H_{2}, H_{2}\right)$ - стационарная корреляционная функция (см. формулу (3) в статье [1]). В соответствии с флуктуационно-диссипативной теоремой [7] дисперсия внутренней энергии КЧ в стационарном случае $\sigma_{2}^{\text {ss }}=\left(H_{2}, H_{2}\right)$ удовлетворяет уравнению

$$
2 \sigma_{2}^{\mathrm{ss}} h(X)=-\gamma\left(F_{21}\right)
$$

Поскольку величины $h(X)(23)$ и $\gamma\left(F_{21}\right)(21)$ известны, то $(25)$ представляет собой уравнение, которое может быть решено относительно дисперсии $\sigma_{2}^{\mathrm{sS}}$. Отметим, что иная ситуация в теории Онзагера, где теорема (25) используется для получения явного вида ковариации случайного члена. Теперь найдем дисперсию внутренней энергии на основе $(25)$ и найденное значение $\sigma_{2}^{\text {sS }}$ подставим в (24). Далее, используя $(21)$ и $(23)$, получим трансцендентное уравнение для $1 / F_{21}$,

$$
\begin{gathered}
F_{21}=\beta_{1}\left\{1-\tilde{a}_{1}\left(F_{21}\right)\right\}^{\frac{1}{2}} \\
\tilde{a}_{1}\left(F_{21}\right)={ }^{*} \tilde{b}\left(\frac{d N_{2}}{d t}\right)\left[f_{4}\left(\frac{\bar{\omega}}{2} X\right)\right]^{-1}, \quad \tilde{b}=\frac{\bar{\varepsilon}}{\tilde{L}_{\dot{H}_{2} \dot{H}_{2}}} .
\end{gathered}
$$

Характер предположений, сделанных при выводе (26), таков, что величина $\tilde{b}$, вошедшая в $(26 \sigma)$, фактически вычислена в пределе $\left(1 / F_{21}\right) \rightarrow \infty$. Однако нетрудно найти вид $b$ 
при произвольных значениях $1 / F_{21}$. Действительно, в общем случае среднюю энергию КЧ можно вычислить по формуле

$$
\langle\varepsilon\rangle=\int d \varepsilon \varepsilon g(\varepsilon) n^{(2)}(\varepsilon),
$$

где $g(\varepsilon)$ - плотность состояний КЧ. Согласно формуле (20) в работе [1] функция плотности $g(\varepsilon)$ имеет максимум, что вообше характерно для плотности состояний быстрых частиц [6]. Максимуму этой функции соответствует значение $\bar{\varepsilon}(2 \mathrm{a})$. Переход к соотношению статистической теории приводит к появлению в $\langle\varepsilon\rangle$ дополнительного множителя - числа заполнения $n^{(2)}(\varepsilon)$. В результате пик полного распределения по энергиям $g(\varepsilon) n^{(2)}(\varepsilon) d \varepsilon$ сдвигается относительно максимума плотности $g(\varepsilon)$ в область меньших энергий. В свою очередь, среднее $\langle\varepsilon\rangle$ также сдвигается относительно $\bar{\varepsilon}$ в сторону меньших значений, так что в первом приближении имеем

$$
\langle\varepsilon\rangle=\lambda \bar{\varepsilon} \exp \left(-F_{21} \bar{\varepsilon}\right) .
$$

В том же приближении коэффициент переноса запишется в виде

$$
L_{\dot{H}_{2} \dot{H}_{2}}\left(F_{21}\right)=\tilde{L}_{\dot{H}_{2} \dot{H}_{2}} \lambda \exp \left(-F_{21}\langle\varepsilon\rangle\right) .
$$

С использованием полученных выражений величину $\tilde{a}_{1}\left(F_{21}\right)(26 \sigma)$ в широком интервале значений квазитемпературы запишем в виде

$$
b\left(F_{21}\right)=\tilde{b}\left\{\exp \left[F_{21}(\langle\varepsilon\rangle-\bar{\varepsilon})\right]\right\} .
$$

Обобшение дает дополнительный множитель в фигурных скобках, который в пределе $\left(1 / F_{21}\right) \rightarrow \infty$ равен единице. Теперь нетрудно обобщить выражение (26б),

$$
a_{1}\left(F_{21}\right)=\stackrel{*}{\varepsilon} b\left(F_{21}\right)\left(\frac{d N_{2}}{d t}\right)\left[f_{4}\left(\frac{\bar{\omega}}{2} X\right)\right]^{-1},
$$

и, заменяя в $(26 \mathrm{a}) \tilde{a}_{1}\left(F_{21}\right)$ на $a_{1}\left(F_{21}\right)$, получить окончательное уравнение для квазитемпературы подсистемы КЧ.

В случае системы с заметным разрывом термодинамических параметров $\left(1 / F_{21}\right) \gg$ $T_{1}$ уравнение (26a) упрошается и сводится к алгебраическому

$$
\begin{aligned}
& \left(x^{2}-1\right) f_{7}\left(x ; \frac{\bar{\omega}}{2} \beta_{1}\right)+a_{2}\left(\beta_{1}\right)=0, \\
& a_{2}\left(\beta_{1}\right)=\stackrel{*}{\varepsilon} \tilde{b}\left(\frac{d N_{2}}{d t}\right)\left[f_{4}\left(\frac{\bar{\omega}}{2} \beta_{1}\right)\right]^{-1},
\end{aligned}
$$

где

$$
x=\frac{F_{21}}{\beta_{1}}, \quad f_{7}\left(x ; \frac{\bar{\omega}}{2} \beta_{1}\right)=(1-x)\left\{1-\left(\frac{\bar{\omega}}{2} \beta_{1}\right)^{2} x\right\}^{-1} .
$$


В (27а) входит произведение двух функций: медленно меняющейся функции $f_{7}\left(x ;(\bar{\omega} / 2) \beta_{1}\right)$ и быстро меняюшейся функции $\left(x^{2}-1\right)$. Воспользуемся приемом, который обычно применяется в аналогичных случаях, а именно, зафиксируем слабоменяющуюся функцию. С этой целью обратим внимание на параметрическую зависимость $f_{7}$ от $(\bar{\omega} / 2) \beta_{1}$. В большинстве реальных ситуаций твердые тела облучаются при комнатных (или близких к комнатным) температурах. Учитывая характерные значения $\bar{\omega}$ на релаксационном этапе каскада, можно установить, что параметр $(\bar{\omega} / 2) \beta_{1}$ меняется в узком интервале вблизи единицы. Если для простоты принять, что $(\bar{\omega} / 2) \beta_{1}=1$, то $f_{7}=1$. Вводя это значение $f_{7}$ в уравнение $(27 \mathrm{a})$, находим аналитическое решение этого уравнения

$$
\frac{1}{F_{21}}=\frac{T_{1}}{\sqrt{1-K^{*} \frac{M}{m}\left(\frac{d N_{2}}{d t}\right)\left[f_{4}\left(\frac{\bar{\omega}}{2} \beta_{1}\right)\right]^{-1}}} .
$$

Величина $K$, вошедшая в (28а), принимает два значения:

$$
K=\left\{\begin{array}{cc}
C_{6} \frac{m a_{\mathrm{B}}^{2}}{Z_{1}^{2} E_{\mathrm{F}} I_{2}(\nu)} & \text { (ферми-газ высокой плотности), } \\
C_{7} \frac{\zeta_{\mathrm{c}}}{(\bar{\varepsilon})^{\frac{3}{2}} E_{\mathrm{F}}^{\frac{1}{2}}\left(\frac{1}{\tau_{\mathrm{p}}}\right)} & \text { (электронньй газ низкой плотности). }
\end{array}\right.
$$

Величина (28б) вычислена с учетом коэффициента переноса $\tilde{L}_{\dot{H}_{2} \dot{H}_{2}}(16),(17)$, в нее вошел численный коэффициент $C_{6}=10^{2}$ и $a_{\mathrm{B}}-$ радиус Бора; она применяется в случае металлов с высокой плотностью электронов $\left(\sim 10^{23} \mathrm{~cm}^{-3}\right)$. Величина $(28 \mathrm{~B})$ вычислена с учетом коэффициента переноса (19), в нее вошел численный коэффициент $C_{7}=0.14$; она применяется в случае невырожденных полупроводников $n$-типа. Наступлению вырождения электронного газа в полупроводниках способствуют малые значения $\zeta_{c}$ и большие концентрации доноров. Поскольку концентрация $N_{\mathrm{d}}$ не зависит от температуры $T_{1}$, величина $K$ также не является функцией температуры. Формулами (28а) и (28в) надлежит пользоваться при плотностях доноров вплоть до $\left(10^{16}-10^{17}\right) \mathrm{cm}^{-3}$, так как при бо́льших концентрациях полупроводники уже считаются вырожденными [12].

Итак, формулы (28) дают искомые выражения для квазитемпературы подсистемы КЧ в состоянии квазиравновесия. Подчеркнем, что вывод этих формул сделан в предположении, что температура термостата заметно ниже квазитемпературы КЧ.

\section{6. ЗАКЛЮЧЕНИЕ}

Обсудим полученные результаты.

6.1. Связь квазитемпературы подсистемы КЧ с температурой термостата. Во всех опубликованных работах по проблеме каскадообразования (см., например, [14]-[16]) температура КЧ $T_{\mathrm{cp}}$ (или в терминологии работ [16], [17] температура теплового пика) определялась с помошью соотношения, взятого из теории равномерного распределения энергии по степеням свободы. Согласно термодинамике классических систем это соотношение в применении к одноатомному газу КЧ имеет вид

$$
T_{\mathrm{cp}}=\frac{2}{3}\langle\varepsilon\rangle .
$$


Однако определение (29) все еше остается достаточно сложным, и в него вносится дополнительное упрошение: средняя энергия $\langle\varepsilon\rangle$ заменяется на характерную энергию $\Theta$ [14]. Выбор величины $\Theta$ весьма произволен. Так, в работе [15] рассматриваются два варианта выбора: либо использовать в качестве $\Theta$ пороговую энергию смешения $\varepsilon_{\mathrm{d}}$, либо величину $(1 / 2) M v_{\mathrm{s}}^{2}$, где $v_{\mathrm{s}}$ - скорость звука. Нам представляется, что полученное таким образом соотношение следует рассматривать всего лишь как эвристическую попытку "угадывания" результата. Оно, разумеется, совершенно неудовлетворительно с логической точки зрения, поскольку теорема о равнораспределении и определение (29) сформулированы для системы в условиях статистического равновесия. Поэтому соотношение (29) неприменимо в условиях каскадного процесса, когда полная система сильно отклонена от равновесия.

Предложенный нами анализ основан на методе статистических ансамблей Гиббса для неравновесных систем. Основой его является неравновесный статистический функционал (см. формулу (12) в статье [1]), на базе которого построено уравнение баланса энергии (1), а затем получено выражение для квазитемпературы (28). В отличие от (29) квазитемпература (28) не связана прямой пропорциональной зависимостью со средним значением энергии, хотя имеется слабая зависимость от $\bar{\varepsilon}$ в случае невырожденного электронного газа (28в). Более того, квазитемпература подсистемы КЧ согласно (28a) пропорциональна температуре $T_{1}$ и в этом смысле может рассматриваться как перенормированная температура термостата. Указанная пропорциональность $T_{1}$ вообще характерна для квазитемпературы, полученной методами неравновесной статистической термодинамики [6], [7], [13].

6.2. Термодинамическая устойчивость стационарных состояний КЧ. Неравновесное стационарное состояние является устойчивым, если все собственные значения матрицы релаксации имеют отрицательные вещественные части [7]. В нашем случае имеется единственное собственное значение (23), так что условие устойчивости принимает вид $h(X)<0$. Последнее, в свою очередь, приводит к условию $a_{1}\left(F_{21}\right)<1$, если в качестве отправной точки взять уравнение (26a), или к условию $a_{2}\left(\beta_{1}\right)<1$, если исходить из уравнения $(27 \mathrm{a})$. Условие, налагаемое на $a_{1}\left(F_{21}\right)$, определяет устойчивость в широком интервале значений квазитемпературы $1 / F_{21}$. В этом случае ее можно проанализировать более подробно. С этой целью решим уравнение баланса (1) при $\left(d \bar{E}_{2} / d t\right)=0$ относительно $\widetilde{\Phi}$, а затем выразим величину $\widetilde{\Phi} / B_{2}$, где

$$
B_{2}=2 L_{\dot{H}_{2} \dot{H}_{2}}\left(F_{21}\right)\left\{\langle\varepsilon\rangle \bar{\omega} N_{1} \sigma^{(\mathrm{d})} \bar{\nu}\left[1-\frac{8}{3} \sigma^{(\mathrm{r})} N_{\mathrm{V}} a\right]\right\}^{-1},
$$

как функцию квазитемпературы $1 / F_{21}$.

График функции $\widetilde{\Phi} / B_{2}$ при различных температурах термостата $T_{1}$ представлен на рисунке. Теоретические кривые получены при $\bar{\omega}=10^{-1}$ эВ, что соответствует оценке характерной энергии на релаксационном этапе (хотя кривые построены для $\bar{\varepsilon}=1 \ni \mathrm{B}$, качественно картина сохраняется и при других $\bar{\varepsilon}$ ). В зависимости от того, каковы значения температуры $T_{1}$, в системе, как показано на рисунке, могут реализоваться стационарные состояния двух типов: с одной квазитемпературой или с тремя значениями 


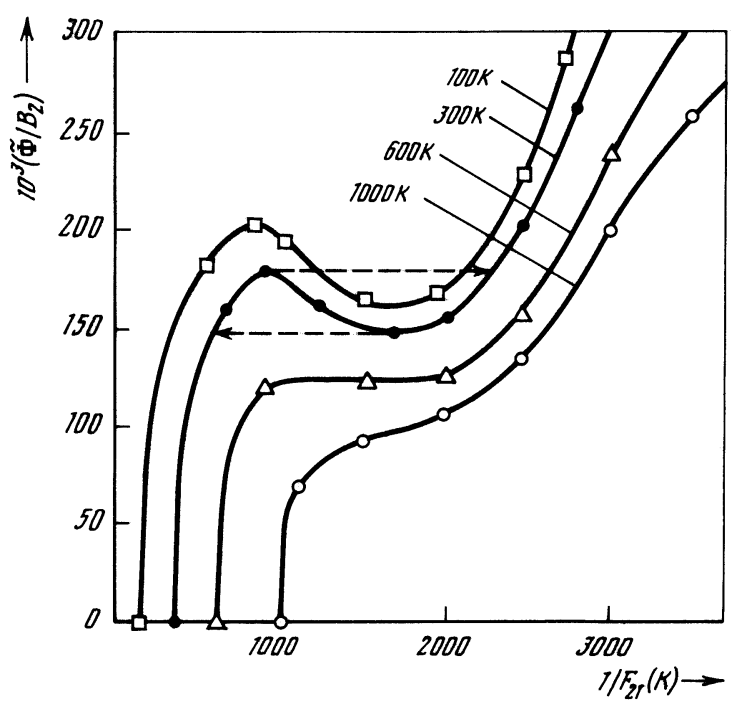

квазитемпературы. Изотерма $T_{1}=600 \mathrm{~K}$ является граничной, разделяюшей изотермы, соответствующие единственному стационарному состоянию, и изотермы, имеющие область кратных стационарных состояний. Если $T_{1}>600 \mathrm{~K}$ и температура термостата поддерживается постоянной, то при каждом значении плотности потока $\widetilde{\Phi}$ существует единственное неравновесное стационарное состояние подсистемы КЧ. В этом состоянии скорость поступления энергии от излучения уравновешивается потерями, связанными с теплопередачей. Напротив, при $T_{1}<600 \mathrm{~K}$ состояния, лежашие между минимумом и максимумом кривой $\widetilde{\Phi} / B_{2}$, неустойчивы. Естественно, в условиях неустойчивости возможны гистерезисные явления, в частности, на изотерме $T_{1}=300 \mathrm{~K}$ стрелками показан гистерезис, наблюдаемый при переходе в область множественных стационарных состояний.

\section{3. Квазитемпература подсистемы КЧ в случае каскада смещений в ме-}

таллах. При вычислении квазитемпературы $1 / F_{21}(28)$ мы учли только один механизм диссипации энергии - отвод энергии из каскадной зоны за счет возбуждения каскадными частицами электронов. Причем на релаксационном этапе, когда скорости КЧ малы, принималось во внимание только возбуждение электронов вблизи поверхности Ферми. Насколько реальна такая ситуация, можно выяснить только в условиях конкретного эксперимента. В этой работе мы придерживались результата анализа из работы [17], согласно которому в случае, когда нейтронами облучаются легкие металлы $\left(Z_{1} \sim 10\right)$, действительно можно ограничиться учетом электронного рассеяния. Используя соотношения (26б) или (27б), нетрудно показать, что условие термодинамической устойчивости выполняется. Значения квазитемпературы подсистемы КЧ при типичных бомбардировках нейтронами $\left(k_{1}=10^{8}\right.$ смешений/атом·c) легких металлов вычислены по 
формулам (28a), (28б) и приведены в таблице.

\begin{tabular}{|c|c|c|c|c|c|c|}
\hline элемент & $Z_{1}$ & $N_{1}, \mathrm{~cm}^{-3}$ & $\frac{d N_{2}}{d t}, \mathrm{c}^{-1}$ & $k_{\mathrm{F}}, \mathrm{cm}^{-1}$ & $\omega_{\mathrm{pl}}$, эВ & $\frac{1}{F_{21}}, \mathrm{~K}$ \\
\hline магний & 12 & $4.3 \cdot 10^{22}$ & $4 \cdot 10^{14}$ & $1.36 \cdot 10^{8}$ & 11 & 950 \\
алюминий & 13 & $6.0 \cdot 10^{22}$ & $6 \cdot 10^{14}$ & $1.75 \cdot 10^{8}$ & 16 & 650 \\
\hline
\end{tabular}

Предполагалось, что облучение металлов происходит при температуре $T_{1}=300 \mathrm{~K}$. Отметим в заключение, что значения $1 / F_{21}$, представленные в таблице, согласуются с оценкой $T_{\mathrm{cp}} \simeq 10^{3} \mathrm{~K}$, которая была дана в ряде экспериментальных и теоретических исследований [15], [17]-[19].

6.4. Квазитемпература в условиях каскада смещений в полупроводниках. Иначе складывается физическая ситуация в случае полупроводников, когда условие термодинамической устойчивости не выполняется. Это связано с малой эффективностью канала отвода энергии, что обусловлено, с одной стороны, низкой плотностью электронов $\left(10^{16}-10^{17} \mathrm{~cm}^{-3}\right)$, а с другой - сильным экранируюшим эффектом $\left(\tilde{I}_{2}(\nu) \sim\right.$ $10^{-2}$ ). Здесь необходим учет еше одного механизма диссипации энергии, обусловленного ядерным рассеянием, точнее, рассеянием КЧ на акустическом потенциале [12]. Последовательный учет этого механизма вносит в теорию большие осложнения, поэтому здесь мы ограничимся кратким обсуждением вопроса. В качестве отправной точки принимается тот факт, что рассеяние на длинноволновых акустических фононах практически является упругим. В области малых скоростей КЧ к столкновениям в каскаде смешений применим степенной потенциал Линдхарда [16], обратно пропорциональный квадрату расстояния. Последнему соответствует полное сечение рассеяния, не зависящее от скорости частицы,

$$
S_{\Pi}=C_{8} Z_{1}^{\frac{5}{3}} e^{2} a,
$$

где $C_{8}=1.3$. Зная $S_{\text {п }}$, нетрудно получить выражение для времени релаксации импульса за счет упругого ядерного рассеяния

$$
\left(\frac{1}{\tau_{\mathrm{p}}}\right)_{\text {ion }}=N_{1} S_{\mathrm{n}} \frac{1}{M v(\bar{\varepsilon})}=C_{8} Z_{1}^{\frac{5}{3}} e^{2} \frac{N_{1} a}{M v(\bar{\varepsilon})}
$$

Как следует из полученного соотношения, в случае резерфордовских столкновений КЧ

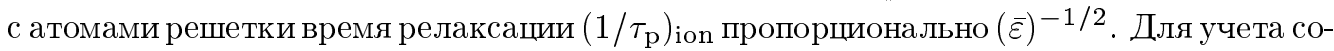
вместного действия электронного и ядерного рассеяния $\mathrm{KЧ} \mathrm{достаточно} \mathrm{заменить} 1 / \tau_{\mathrm{p}}$ в $(28$ в $)$ на $\left(1 / \tau_{\mathrm{p}}\right)_{\mathrm{e}}+\left(1 / \tau_{\mathrm{p}}\right)_{\text {ion. }}$. Принимая во внимание формулы $(26 б),(19)$ и $(30)$, для кремния $n$-типа при температуре $T_{1}=300 \mathrm{~K}$ находим $a_{1}\left(F_{21}\right) \simeq 0.2$. Таким образом, при одновременном учете двух видов рассеяния условие устойчивости состояния КЧ выполняется, а квазитемпература подсистемы КЧ превышает температуру решетки полупроводника. 


\section{Список литературы}

[1] Ю. А. Кашлев. ТМФ. 2000. Т. 123. № 3. С. 485.

[2] Я. С. Дербенев. Теория электронного охлаждения. Автореф. дис. на соис. уч. ст. д.ф.-м.н. Новосибирск: Институт ядерной физики, 1978.

[3] H. Ikari. J. Phys. Soc. Japan. 1979. V. 46. № 1. P. 97.

[4] T. McMullen. J. Phys. 1977. V. 7. № 4. P. 2041.

[5] П. С. Зырянов, М.И. Клингер. Квантовая теория электронного переноса в кристаллических полупроводниках. М.: Наука, 1976.

[6] Yu. A. Kashlev. Phys. Stat. Sol. B. 1995. V. 190. P. 379.

[7] Дж. Кайзер. Статистическая термодинамика неравновесных процессов. М.: Мир, 1990.

[8] М. Гольдман. Спиновая температура и ЯМР в твердых телах. М.: Мир, 1972.

[9] Ю. А. Кашлев. ТМФ. 1995. Т. 102. № 1. С. 106.

[10] Л. Каданов, Г. Бейм. Квантовая статистическая механика. Методы функций Грина в теории равновесных и неравновесных процессов. М.: Мир, 1964.

[11] J. Hubbard. Proc. Roy. Soc. A. 1958. V. 243. P. 336.

[12] К. Зеегер. Физика полупроводников. М.: Мир, 1977.

[13] E. C. Zimmerman, J. Ross. J. Chem. Phys. 1984. V. 80. P. 720.

[14] P. Sigmund. J. Appl. Phys. 1974. V. 25. № 3. P. 170.

[15] V. I. Protasov, V. G. Chudinov. Rad. Eff. 1982. V. 66. № 1. P. 1.

[16] К. Лейман. Взаимодействие излучения с твердым телом. М.: Атомиздат, 1979.

[17] F. Sietz, J. S. Koehler. Solid State Phys. 1956. V. 2. P. 305.

[18] Д. Динс, Д. Винйард. Радиационные эффекты в твердых телах. М.: ИЛ, 1960.

[19] R.S. Nelson. Phil. Mag. 1965. V. 11. P. 291.

Поступила в редакцию 6.VI.2000 г., после доработки 4.IX.2000 г. 